\title{
NETWORK-CENTRIC TECHNOLOGIES FOR CONTROL OF THREE-PHASE NETWORK OPERATION MODES
}

\begin{abstract}
Purpose. The development of the control system for three-phase network is based on intelligent technologies of network-centric control of heterogeneous objects. The introduction of unmanned aerial vehicles for monitoring of three-phase network increases the efficiency of management. Methodology. The case of decomposition of the instantaneous capacities of the fixed and variable components for 3-wire system. The features of power balance for the different modes of its functioning. It should be noted that symmetric sinusoidal mode is balanced and good, but really unbalanced, if the standard reactive power is not zero. To solve the problem of compensation is sufficient knowledge of the total value of the inactive components of full power (value of the inactive power) without detail. The creation of a methodology of measurement and assessment will require knowledge of the magnitudes of each inactive component separately, which leads to the development of a unified approach to the measurement and compensation of inactive components of full power and the development of a generalized theory of power. Results. Procedure for the compensation of the current of zero sequence excludes from circuit the source, as the active component of instantaneous power of zero sequence, and a vector due to a current of zero sequence. This procedure is performed without time delay as it does not require integration. Only a 3-wire system with symmetrical voltage eliminates pulsations and symmetrization of the equivalent conductances of the phases of the task. Under asymmetric voltage, the power is different, its analysis requires the creation of a vector mathematical model of the energy processes of asymmetrical modes of 3-phase systems. Originality. The proposed method extends the basis of the vector method for any zero sequence voltages and shows that the various theories of instantaneous power three wired scheme due to the choice of a basis in a two-dimensional subspace. Practical value. The algorithm and software implementation for the decomposition of the zero sequence current, which allocated the procedure of obtaining null-balanced vectors of phase and interfacial voltage, calculation of active and inactive instantaneous power is zero balanced mode. The simulation results obtained in the software package Matlab by the method of visual programming in Simulink. References 9, figures 5.
\end{abstract}

Key words: network-centric control, unmanned aerial vehicle, losses, quality, monitoring, instantaneous power.

Интеграция интеллектуальных и сетецентрических технологий в процесс управления режимами работы трехфазной сети обеспечивают оперативность компенсации нелинейностей в системе за счет ортогонального разложения тока и использования метода базисных функций для минимизации потерь. Библ. 9, рис. 5.

Ключевые слова: сетецентрическое управление, беспилотный летательный аппарат, потери, качество, мониторинг, мгновенная мощность.

Introduction and problem definition. System processes of intellectualization of Ukrainian electric power system are based on the introduction of Smart Grid technologies. The value of this technological transformation is to redistribute the demand for electric power at the moments of maximum load, to reduce additional investments in the reorganization of the power system in order to increase its productivity [1]. Multilevel development of the control system of the operation modes of a three-phase network implies the use of the principle of network-centricity - the control of heterogeneous objects of the energy system infrastructure in a single information and communication management space due to the formation and maintenance of a single integrated contextual information environment for all tiers [2].

The concept of network-centric control of the modes of operation of a three-phase network implies the formation and maintenance in the actual state of a single image of the real state in the most understandable and simple form for the whole system. One of the ways to achieve these management objectives is to introduce into the system of operational maintenance of the power system [1], in addition to the distributed subsystem of digital measuring modules, a group of unmanned aerial vehicles (UAV) for monitoring the state of a three-phase network [3]. Multi-agent technologies for the collection and transmission of information using UAVs ensure the continuity of acquisition and the relevance of the contextual information image of the three-phase network.
Reactivity, asymmetry and nonlinearity of load in a three-phase system lead to the presence of inactive components of the total power and causes not only additional losses of electricity, but also causes the appearance of pulsations of instantaneous power (IP) the energy non-equilibrium of the system. This causes a decrease in the efficiency, it contributes to the occurrence of dangerous resonance phenomena during the operation of the equipment.

Analysis of recent investigations and publications. The efficiency of the use of electric energy is determined mainly by the creation of such conditions for its consumption, under which the required quality of electric energy supply is provided with minimal losses $[4,5]$. The quality of electricity supply can significantly affect the power consumption, reliability of the power supply system [6]. Exceed of the quality indicators of electric energy above the permissible leads to a reduction in the service life of the equipment, a decrease in its efficiency and a violation of the technological process. Minimization of losses in the 3-phase system is significantly associated with the possibility of reducing additional losses that are caused by consumption nodes with asymmetric and non-linear loads [7].

The goal of investigations is the development of methods for compensating the inactive component of instantaneous power in the presence of an asymmetric load under conditions of network-centric control of the operating modes of a three-phase network.

(C) Y.I. Sokol, Yu.A. Sirotin, T.S. Iierusalimova, O.G. Gryb, S.V. Shvets, D.A. Gapon 
Main materials of investigations. A 3-wire circuit is a special case of the 4 -wire circuit. The introduced definitions of unbalanced (balanced, really balanced) and unbalanced (balanced) modes remain valid for the 3-wire scheme. However, IP of 0 -balanced processes are used to classify modes.

In a 3-wire circuit:

- the active instantaneous power is fully determined by 0 -balanced current and voltage processes

$$
p(t)=p_{!}(t)=i_{!}^{\bullet} u_{!},
$$

- the vector IP coincides with its 0 -component

$$
q(t)=q_{0}(t)=q_{0}(t) e_{0}
$$

and is fully determined by $q_{0}=q_{0}(t)=[i \times u]^{\bullet} e_{0}$ algebraic projection of the vector IP onto the unit vector $e_{0}$ which we call a scalar inactive IP.

The decomposition of instantaneous powers into constant and variable components for:

- active IP:

$$
\bar{p}_{!}=\frac{1}{T} \int_{\tau}^{\tau+T} p_{!}(t) d t ; \quad p_{!}(t)=\bar{p}_{!}+\tilde{p}_{!}(t),
$$

where $\tau \geq 0$ is the arbitrary number;

- inactive (scalar) IP:

$$
\bar{q}_{0}=\frac{1}{T} \int_{\tau}^{\tau+T} q_{0}(t) d t ; \quad \tilde{q}_{0}(t)=q_{0}(t)-\bar{q}_{0},
$$

classify processes in a 3-wire circuit.

If active IP has no variable (pulsed) component $\tilde{p}_{!}(t) \equiv 0$, then mode is balanced. In the general caseB общем случае $\tilde{p}=p(t)-\bar{p} \neq 0$ and the mode is unbalanced.

As $q(t)=q_{0}(t)=q_{0}(t) e_{0}$, then the mode:

- at which inactive IP has no variable component $\widetilde{q}_{0}=\widetilde{q}_{0}(t) \equiv 0$ is balanced;

- at which the inactive IP is identically equal to zero $q_{0}(t) \equiv 0$ is really balanced.

Note that the symmetric sinusoidal mode is balanced and balanced, but it is really unbalanced if the standard reactive power is not zero.

Decomposition of current in a 4-wire network with the isolation of zero sequence (ZS). For a 4-wire circuit, the decomposition of the current $i=i_{0}+i_{\text {! }}$ is valid The basis curves $w_{1}(t), w_{2}(t)$ of processes in 3-wire circuit which are used for the decomposition of the current components, are complemented by the unit vector of the ZS.

The voltage vector $u$ (measured with respect to an arbitrary reference point) defines two orthogonal 0-balanced vectors:

- the vector of phase voltages (using a projector matrix $\left.u_{!}=D_{!} \times u\right)$ and

- the vector of phase-to-phase voltages (using a skewsymmetric matrix $u_{\|}=K^{\bullet} \times u_{!}$).

At each moment of time, the triple of vectors: $w_{1}(t)$, $w_{2}(t), e_{0}$ forms an orthonormal basis of the space $S^{(3)}$, since the following orthonormality condition is valid: $\left[\begin{array}{l}w_{1}^{\bullet} \\ w_{2}^{\bullet} \\ - \\ e_{0}^{\bullet}\end{array}\right] \times\left[w_{1} w_{2} \mid e_{0}\right]=\left[\begin{array}{ccc}w_{1}^{\bullet} w_{1} & w_{1}^{\bullet} w_{2} & w_{1}^{\bullet} e_{0} \\ w_{2}^{\bullet} w_{1} & w_{2}^{\bullet} w_{2} & w_{2}^{\bullet} e_{0} \\ e_{0}^{\bullet} w_{1} & e_{0}^{\bullet} w_{2} & e_{0}^{\bullet} e_{0}\end{array}\right]=\left[\begin{array}{ccc}1 & 0 & 0 \\ 0 & 1 & 0 \\ 0 & 0 & 1\end{array}\right]$.

The orthogonal current decomposition is valid:

$i(t)=\underbrace{i_{1} w_{1}+i_{2} w_{2}}_{i}+\underbrace{i_{0} w_{0}}_{i_{0}}=\underbrace{\left(i^{\bullet} w_{1}\right.}_{i_{1}}) w_{1}+\underbrace{\left(i^{\bullet} w_{2}\right.}_{i_{2}}) w_{2}+i_{0}(t) e_{0}$

because $i=i_{1}+i_{0}, \quad$ and $i_{0} \perp u_{!} ; \quad i_{0} \perp u_{\|}, \quad$ then for the decomposition (4) coefficients is valid the following:

$$
\begin{gathered}
i_{1}=i^{\bullet} w_{1}=\left(i_{!}+i_{0}\right)^{\bullet} \times \frac{u_{!}}{\left|u_{!}\right|}=\frac{i_{!}^{\bullet} u_{!}}{\left|u_{!}\right|}=\frac{p_{!}}{\left|u_{!}\right|} ; \\
i_{2}=i^{\bullet} w_{2}=\left(i_{!}+i_{0}\right)^{\bullet} \times \frac{u_{\|} \mid}{\left|u_{\|}\right|}=\frac{i_{!}^{\bullet}\left(u_{!} \times e_{0}\right)}{\left|u_{!}\right|}= \\
=\frac{e_{0}\left(i_{!} \times u_{!}\right)^{\bullet}}{\left|u_{!}\right|}=\frac{q_{0}(t)}{\left|u_{!}\right|} \\
i_{0}(t)=i^{\bullet} e_{0} .
\end{gathered}
$$

Therefore, the decomposition (4) is obtained from the decomposition for the 3-wire circuit

$$
i_{0}=i_{1}+i_{2}=\frac{p_{!}(t)}{u_{!}} w_{!}+\frac{q_{0}(t)}{u_{!}} w_{\|},
$$

by additional term (7) . This gives decomposition of the current for a 4-wire circuit (Fig. 1) in vector-matrix form:

$$
i(t)=i_{1}+i_{2}+i_{0}=\left[\begin{array}{lll}
w_{!} & w_{\|} & e_{0}
\end{array}\right] \times\left[\begin{array}{c}
p_{!}(t) / u_{!} \\
q_{0}(t) / u_{!} \\
i_{0}(t)
\end{array}\right],
$$

$$
u_{!} \neq 0 \text {. }
$$

Decomposition is valid at any voltage $u_{0}(t)$ of 0 -sequence.

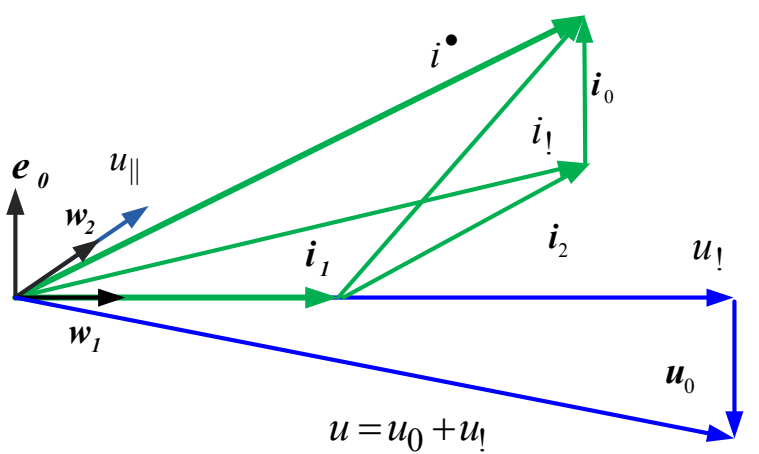

Fig. 1. Current and voltage decomposition in the 4-wire system $\left(u_{0} \| e_{0}\right)$

As $p_{0}=u_{0} \cdot i_{0}$, then compensation of current of 0 -sequence always compensate power of 0 -sequence $\left(i_{0}=0 \Rightarrow p_{0}=0\right)$. The converse is not true. In addition, the part of the vector IP is also compensated.

The proposed basis method expands the vector method $[8,9]$ for any ZS voltage $u_{0}(t)$ and shows that the different theories of the IP of the 3 -wire circuit are due to the choice of basis in the 2-dimensional subspace $L_{!}^{2}$.

Features of compensation by the method of basis functions. Before the compensation, the current in the 
load network is equal to the current of the source $i_{S}(t)=i_{L}(t)=i(t)$ and can contain the 0 -sequence current regardless of the presence $\left(u_{0} \neq 0\right)$ or the absence $\left(u_{0}=0\right)$ of the voltage displacement.

Compensation of the current of the 0 -sequence $i_{0}$ excludes from the source circuit:

- the active IP of the 0 -sequence (both its constant and the variable components at any 0 -sequence voltage);

- the vector IP component due to the ZS.

Moreover, this procedure is performed without delay in time, since it does not require integration. Integration and compensation of the 0 -balanced current component $i_{2}$ (collinear phase-to-phase voltage) which determines the inactive IP, due to 0-balanced current and voltage processes, does not require integration. Compensation of currents $i_{0}(t)$ and $i_{2}(t)$ is equivalent to compensation of the vector IP and 0 -component of active power (if $u_{0} \neq 0$ ) and is carried out without delay in time. Compensation of active current:

$$
\tilde{i}_{1}=\frac{\tilde{p}_{!}(t)}{u_{!}} w_{!}=\left(p_{!}(t)-P_{!}\right) \frac{u_{!}}{u_{!}^{2}}
$$

is associated with the pulsating component of the active IP and will require integration. In addition, the expansion coefficients determine the connection of the current vector in the $\alpha \beta$ coordinates of the orthonormal basis in the 2-dimensional subspace and instantaneous powers and can be found without intermediate calculations directly using the measured instantaneous currents $i(t)$ and instantaneous stress values $u(t)$.

Algorithm and implementation of the current decomposition program

$$
i(t)=\frac{p_{!}(t)}{u_{!}(t)} w_{!}(t)+\frac{q_{0}(t)}{u_{!}(t)} w_{\|}(t)+i_{0}(t) e_{0}
$$

ss determined by the following procedures:

- calculation of 0 -balanced vector of phase and phaseto-phase voltage:

$$
u_{!}(t)=D_{!} u(t), \quad u_{\|}(t)=K^{\bullet} u(t) ;
$$

- calculation of active IP and inactive IP of 0-balanced mode:

$$
\begin{gathered}
p_{!}(t)=i^{\bullet} u_{!}=i^{\bullet} D_{!} u, \\
q_{0}(t)=i^{\bullet} u_{\|}=i^{\bullet} K^{\bullet} u .
\end{gathered}
$$

By the Simulink visual programming method the program in the Matlab package is implements. The Matlab package constructs a block diagram of the program using a palette of mathematical model components of various specified electrical power devices. The developed program also implements the current decomposition (15), the first component of which provides power supply with constant power:

$$
i=\frac{P}{u_{!}} w_{!}+\underbrace{\frac{\tilde{p}_{!}(t)}{u_{!}} w_{!}+\frac{q_{0}(t)}{u_{!}} w_{!}+i_{0}(t) e_{0}}_{\text {compensated current }} .
$$

- The simulation results (Fig. 2-5) represent qualitatively the energy processes in the 4-wire circuit of power supply, in particular, confirm (Fig. 2) the theoretically known behavior of the active and inactive current of $p q$-theory in the sine-wave mode with symmetrical voltage: the active current realizing the active IP and the reactive current realizing the vector IP (second and third pallets from above) contain a harmonic of the 3-order.

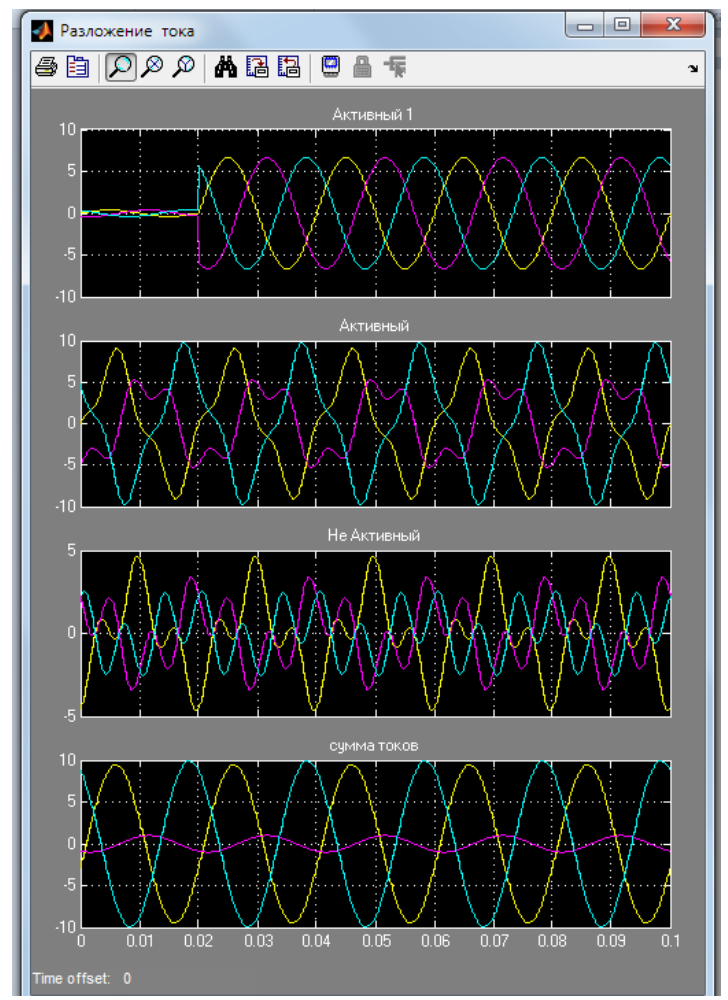

Fig. 2. Oscillograms of total current decomposition onto components

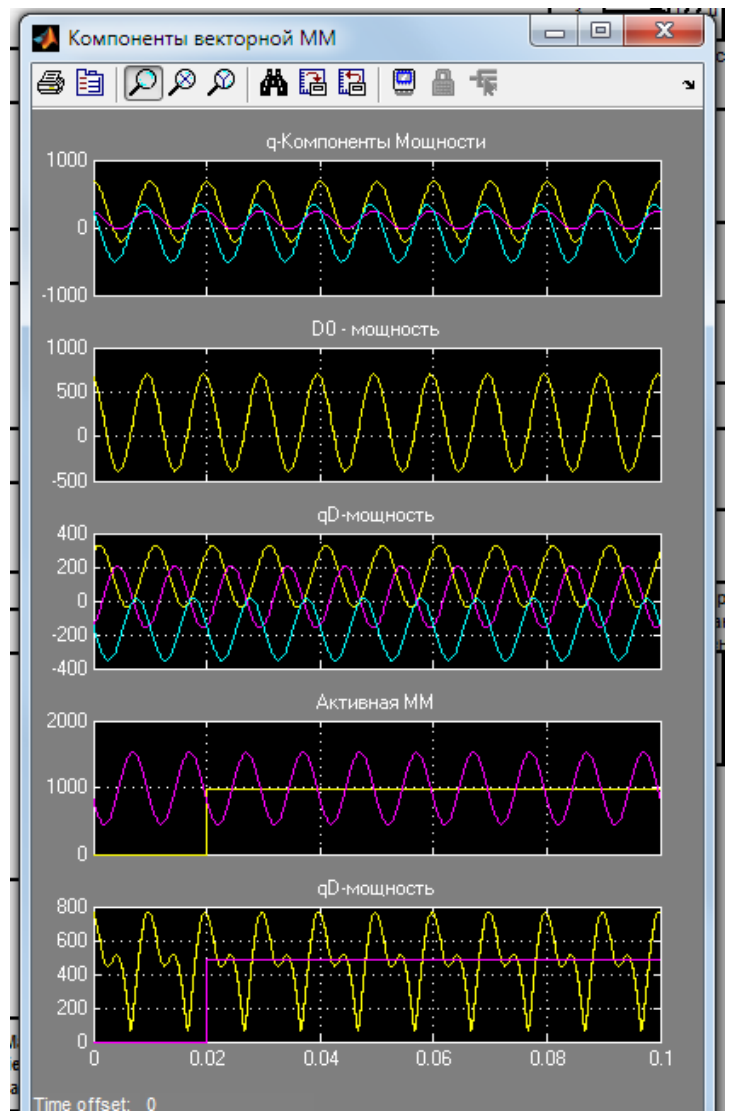

Fig 3. Oscillograms of vector and scalar IP decomposition onto components 


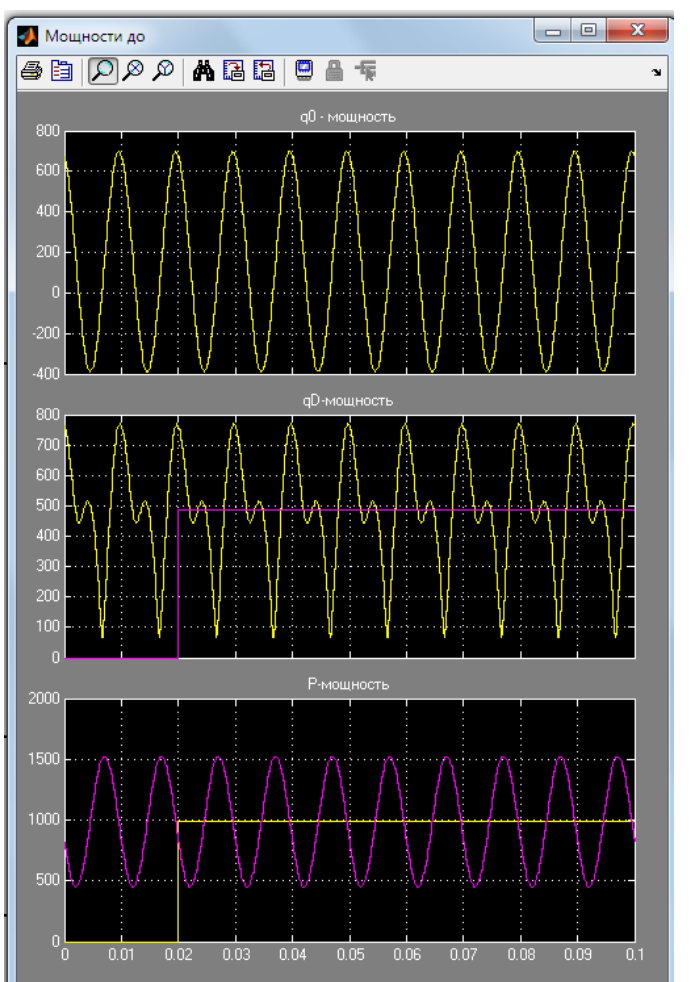

Fig. 4. Oscillograms of scalar IP and components of vector IP before compensation

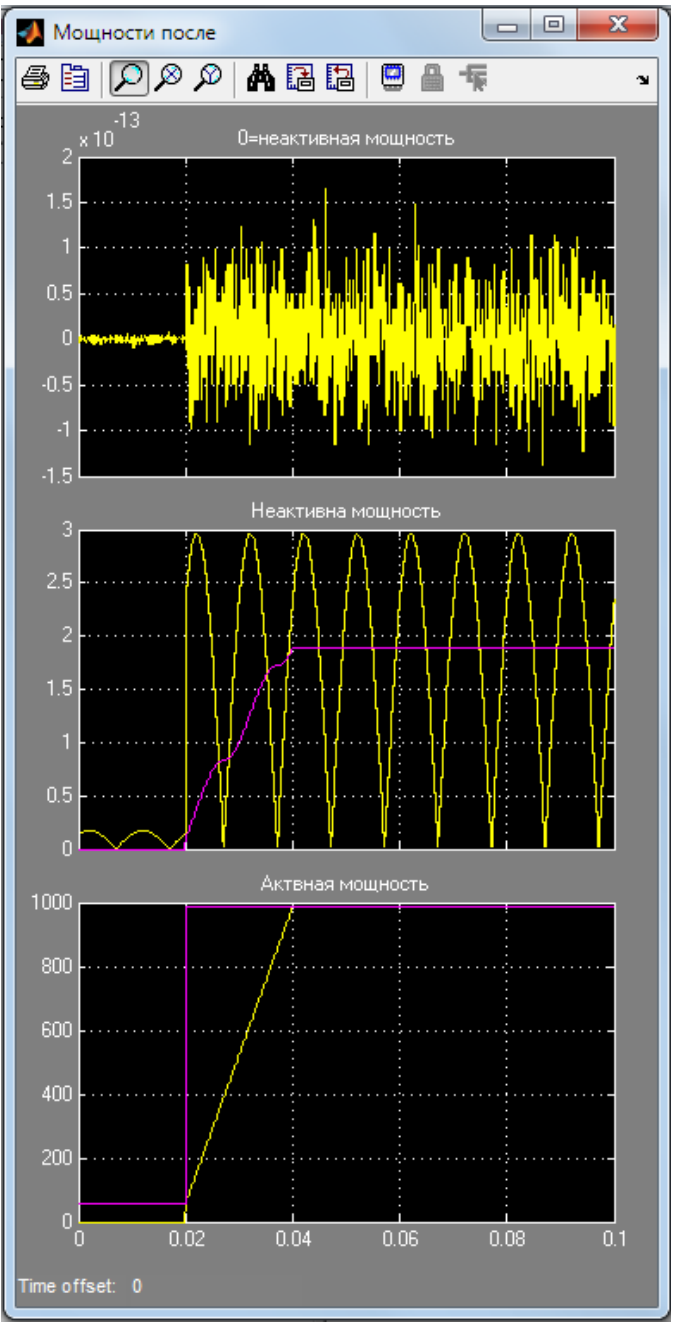

Fig. 5. Oscillograms of scalar IP and components of vector IP after compensation
The active current which realizes the integral active power:

$$
i_{S}=u_{!}\left(\frac{P}{u_{!}^{2}}\right),
$$

(Fig. 5, bottom pallet) and provides a mode of consumption with current in the source circuit at which:

- current is 0 -balanced $i_{0}(t) \equiv 0 \Leftrightarrow q^{\prime}(t) \equiv 0$ (middle pallet in Fig. 5);

- the mode is really balanced $q_{0}(t) \equiv 0$ (top pallet in Fig. 5);

- the mode is balanced $p_{!}(t) \equiv \bar{p}_{!}=P_{!}$(bottom pallet in Fig. 5).

Conclusions. Network-centric technologies for controlling the operating modes of a three-phase network using multi-agent methods for collecting and transmitting information using UAVs ensure the continuity of acquisition and the relevance of the contextual information image of the state of the power system.

The case of the decomposition of instantaneous powers into a constant and a variable component for a 3wire system is considered. The peculiarities of the power balance for different modes of its functioning are singled out. It should be noted that the symmetric sinusoidal mode is balanced, but it is really unbalanced if the standard reactive power is not zero.

The zero sequence current compensation procedure excludes from the source circuit both the active component of the instantaneous power of the zero sequence and the vector component caused by the zero sequence current. This procedure is performed without delay in time, since it does not require integration.

To solve the compensation problem, it is enough to know the total value of the inactive components of the total power (the value of inactive power) without its detailed elaboration. The creation of a measurement and accounting methodology will require knowledge of the values of each inactive component separately, which necessitates the development of a unified approach to measuring and compensating inactive components of full power and developing a generalized theory of power. Only in a 3-wire system with symmetrical voltage, the elimination of pulsations and the symmetrization of phase conductivities are equivalent problems (the power of pulsations and the power of asymmetry of phase conductivities are equal). With unbalanced voltage these powers are different, their analysis for electrical systems requires the creation of a vector mathematical model of the energy processes of asymmetric regimes of 3-phase systems.

Unbalanced loads, which, in addition to additional losses, lead to asymmetry in the voltage and pulsation of the energy flow, cause much greater harm to the power supply than the symmetry of the reactive phase conductivities (reactive power).

\section{REFERENCES}

1. Sokol Y.I., Gryb O.G., Shvets S.V. The structural and parametrical organization of elements of a power supply system in the conditions of network centrism. Electrical engineering \& 
electromechanics, 2016, no.2, pp. 61-64. (Rus). doi: 10.20998/2074-272X.2016.2.11.

2. Sokol Y.I., Gryb O.G., Shvets S.V. Network centrism optimization of expeditious service of elements of the power supply system. Electrical engineering \& electromechanics, 2016, no.3, pp. 67-72. (Rus). doi: 10.20998/2074272X.2016.3.11.

3. Shvets S.V., Voropai U. G. Mariechantal aspects of the use of unmanned aerial vehicles. Bulletin of Kharkiv Petro Vasylenko National Technical University of Agriculture, 2016, no.176, pp. 33-34. (Ukr).

4. Denisyuk S.P. Optimization of power consumption for energy saving in systems with converters. Problems of energy saving, 1994, no.2-3, pp. 81-88. (Rus).

5. Prakhovnik A.V. Energy saving: unconventional look and a different strategy. Energetic and electrification, 2008, no.4, pp. 30-33. (Rus).

6. Shidlovsky A.K., Kuznetsov V.G. Povyshenie kachestva elektroenergii $v$ elektricheskikh setiakh [Increase the power quality in electric networks]. Kyiv, Naukova Dumka Publ., 1985. 286 p. (Rus).

7. Zhezhelenko I.V., Saenko Yu.L. Voprosy kachestva elektroenergii v elektroustanovkakh [Issues of power quality in electrical installations]. Mariupol, PSTU Publ., 1996. 173 p. (Rus).

8. Tenti P., Mattavelli P., Tedeschi E. Compensation techniques based on reactive power conservation. 7 th Int.
Workshop on Power Definitionsand Measurements under NonSinusoidal Conditions, Cagliari, Italy, July 2006, pp. 84-90.

9. Sirotin Yu.A. Unbalanced current and the pulsating current at asymmetrical voltage. Tekhnichna elektrodynamika, 2012, no.2, pp. 42-43.

Received 25.04.2017

Y.I. Sokol ${ }^{1}$, Doctor of Technical Science, Professor,

Corresponding Member of the National Academy of Science of Ukraine,

Yu.A. Sirotin ${ }^{1}$, Doctor of Technical Science, Professor,

T.S. Iierusalimova ${ }^{1}$, Candidate of Technical Science,

O.G. Gryb ${ }^{1}$, Doctor of Technical Science, Professor,

S.V. Shvets ${ }^{1}$, Candidate of Technical Science, Associate

Professor,

D.A. Gapon ${ }^{1}$, Candidate of Technical Science, Associate

Professor,

${ }^{1}$ National Technical University «Kharkiv Polytechnic Institute», 2, Kyrpychova Str., Kharkiv, 61002, Ukraine, phone +38 0577076551 ,

e-mail: Ierusalimovat@mail.ru,dima12345ml@mail.ru, se_sx@bk.ru

How to cite this article:

Koliushko D.G., Rudenko S.S. A computer program for interpretation of the data of vertical electrical sounding VEZ-4A. Electrical engineering \& electromechanics, 2017, no.3, pp. 67-71. doi: 10.20998/2074-272X.2017.3.10. 